\title{
Humoral response against myelin associated glycoprotein reflects oligodendroglial degeneration in Parkinson's disease
}

\author{
Ewa Papuć ${ }^{1}$, Barbara Wilczyńska², Konrad Rejdak ${ }^{1}$ \\ ${ }^{1}$ Department of Neurology, Medical University of Lublin, Poland \\ ${ }^{2}$ Chair and Department of Clinical Immunology, Medical University of Lublin, Poland
}

Papuć E, Wilczyńska B, Rejdak K. Humoral response against myelin associated glycoprotein reflects oligodendroglial degeneration in Parkinson's disease. Ann Agric Environ Med. 2016; 23(2): 390-393. doi: 10.5604/12321966.1203998

\begin{abstract}
Identification of disease-specific diagnostic and prognostic biomarkers which would enable early detection and follow-up of Parkinson's disease (PD) is a crucial problem. Recently, we confirmed the presence of adaptive immune response against different glial-derived antigens in PD.

Objective. The aim of the study was to assess humoral response against myelin-associated glycoprotein (MAG) on a larger group of PD patients. IgM autoantibodies against MAG were measured by an ELISA system in 66 PD patients and 66 control subjects.

Results. The study confirmed a significantly increased production of anti-MAG IgM antibodies in parkinsonian patients $(p<0.05)$. No correlations were found between anti-MAG IgM antibody titers and disease severity measured on the HoehnYahr scale, MMSE or age of PD onset ( $p>0.05$ ). The results provide evidence for activation of humoral response against MAG in PD patients, but argue against the utility of anti-MAG antibodies as biomarkers of disease severity. The results additionally indicate the potential protective role of autoimmunity in maintaining the body's homeostasis, which may involve the clearance of abnormal proteins. Further studies are necessary to confirm the role of anti-MAG antibodies as biomarkers of $\mathrm{PD}$, especially in relation to other neurodegenerative disorders.
\end{abstract}

\section{Key words}

anti-MAGantibodies, humoral response, immune system, glial cells, Parkinson's disease

\section{INTRODUCTION}

Identification of diagnostic and prognostic biomarkers which would allow an early diagnosisand clinical follow-up of patients with different neurodegenerative disorders is an important problem in modern neurology. There is emerging evidence that humoral response may be involved in the pathogenesis and progression of different neurodegenerative disorders, including Parkinson's disease (PD) $[1,2,3]$. Autoantibodies specific to different self-antigens have been found in a variety of neurological disorders $[4,5,6,7]$ as well as in PD $[1,3,8]$. Nevertheless, assessment of the role of antibodies directed against the most important self-antigen in PD, alpha-synuclein, has produced confounding results. Different studies report higher [3,9], comparable [10] or even lower [11] levels of alpha-synuclein antibodies in PD patients, compared with healthy control subjects.

There is increasing evidence that the neurodegenerative process in $\mathrm{PD}$ involves not only degeneration of neuronal structures, but also deterioration of glial cells, as alphasynuclein-positive inclusions have been found not only in neurons, but also in oligodendrocytes and astrocytes of PD subjects $[12,13]$. The presented study is a continuation of the authors' studies on humoral immune response against glial-derived antigens in $\mathrm{PD}$, which is probably a reaction to the neurodegenerative process of the central nervous system.

Address for correspondence: Ewa Papuć, Department of Neurology, Medical University, Jaczewskiego 8, 20-954 Lublin, Poland

e-mail: ewapap@yahoo.pl

Received: 21 March 2016; accepted: 11 May 2016
In a previous study [14], the authors' found significantly higher titers of autoantibodies against different glialderived antigens, including anti-MAG antibodies, in a small group of PD patients, in comparison to healthy subjects. Higher antibody titers against other different myelin antigens, e.g. myelin basic protein (MBP) and myelinoligodendrocyte glycoprotein (MOG), in demented PD patients, and DLB were also previously found by Maetzler et al. [2]. However, these results were not confirmed in a later study conducted on a larger group of PD patients by the same authors [15]. Additionally, in other neurodegenerative disorders (DLB, AD), antibodies against alpha-synuclein [16], and glial-derived antigens [17] have been found to be higher in comparison to controls.In the light of these equivocal data, for the current study it was decided to assess anti-MAG response in PD patients, and to assess the utility of these antibodies as biomarkers of disease severity.

Autoimmune reactions against specific proteins and their self-assembled complexes are probably involved in the disease pathology, and they could therefore potentially be used as sensitive biomarkers of neurodegeneration $[2,18]$. In addition, humoral response may play a role in neuroprotection and in introducing new therapies. Data from models of stroke and traumatic brain injury have revealed that anti-MAG antibodies have a neuroprotective action $[19,20]$. Additionally, in another neurodegenerative disorder, Alzheimer's disease, much attention has recently been focused on vaccine development, including both passive vaccination with antibodies and active vaccination with $\mathrm{Ab} 42$ peptide and its pre-aggregated forms [21]. 
In the presented study, humoral response against myelin-associated glycoprotein (MAG) wasa assessed. It was hypothesized that in PD the immune response may not only be directed against antigens typically present in dopaminergic neurons, but may be a more widespread process directed against antigens of glial cells. MAG is a protein of glial origin, expressed selectively on the innermost part of the myelin sheath, which adheres directly to the axon surface and protects neurons from excitotoxicity. It also regulates interactions between myelin and the axon [18].

\section{MATERIALS AND METHOD}

$66 \mathrm{PD}$ patients in different clinical stages (scores of $1-5$ on the Hoehn-Yahr scale) consecutively admitted to the Department of Neurology of the Medical University of Lublin, Poland, were enrolled for the study. PD patients were diagnosed and assessed on the Hoehn-Yahr scale by specialists in neurodegenerative movement disorders (EP, KR). All patients fulfilled the UKPDS Brain Bank criteria [22]. The group of PD patients was divided into two subgroups: mild/moderate PD: Hoehn-Yahr grade 1-3, n=42; advanced PD: Hoehn-Yahr grade $4-5, n=24$. In addition, serum samples from 66 healthy controls matched for age and gender were assessed. Subjects with blood transfusion, treated with immunomodulatory agents, or with a history of an infection or an inflammatory process three months prior to the study were excluded from the study.

Blood was collected between 08:00 - 10:00, transferred to the lab on ice, and centrifuged; the serum was stored at $-70^{\circ} \mathrm{C}$ within 60 minutes.

The study was approved by the local Ethics Committee, and all participants gave their written informed consent to participate in the study.

IgM autoantibodies against myelin-associated glycoprotein (MAG) were measured by a commercially available ELISA system according to the manufacturer's instructions (BlueGene Biotech).

The titers were estimated on the basisof a calibration curve of autoantibody standards and expressed in nanograms per milliliter (ng/mL). The sensitivity of the assay was $1.0 \mathrm{ng} / \mathrm{mL}$.

Statistical analysis. Differences in antibody titers between the two evaluated groups were estimated using a t-test. Differences between the subgroups of PD patients (advanced versus mild/moderate $\mathrm{PD}$ ) were calculated using the MannWhitney U test. To assess correlations, Spearman's rho correlation coefficients were calculated. $\mathrm{P}$ value $<0.05$ was considered statistically significant (two-sided). Statistical calculations were performed using InStatGraphPad Software Inc, (CA, USA).

\section{RESULTS}

Autoantibodies against MAGwere detected in sera of all the investigated subjects. Significantlyhigher titers of antiMAG IgM autoantibodies were observed in PD patients in comparison to healthy control subjects $(\mathrm{p}=0.003)$. No statistically significant differences were found in anti-MAG antibody titers between advanced PD patients (grade 4-5 on the Hoehn-Yahr scale; median: 1.79) and patients with
Table 1. Demographic data of the study population.

MMSE - Mini Mental State Examination. NA - not applicable. Data are presented as means with standard deviation (SD)

\begin{tabular}{lll}
\hline $\begin{array}{l}\text { Demographic characteristic of study } \\
\text { group }\end{array}$ & PD patients & Control subjects \\
\hline Subjects (female/male) & $\mathrm{n}=66(40 / 26)$ & $\mathrm{n}=66(38 / 28)$ \\
\hline Age [years]mean \pm SD & $\begin{array}{l}64.32 \pm 8.05 \\
(43-81)\end{array}$ & $\begin{array}{l}59.54 \pm 13.80 \\
(26-70)\end{array}$ \\
\hline $\begin{array}{l}\text { Hoehn-Yahr scale } \\
\text { mean } \pm \text { SD (range) }\end{array}$ & $3.02 \pm 1.09(1-5)$ & $\mathrm{NA}$ \\
\hline PD patients with predominant tremor & $46(69.7 \%)$ & $\mathrm{NA}$ \\
\hline $\begin{array}{l}\text { PD patients with predominant axial } \\
\text { features }\end{array}$ & $20(30.3 \%)$ & $\mathrm{NA}$ \\
\hline $\begin{array}{l}\text { Age of PD onset [years] } \\
\text { mean } \pm S D \text { (range) }\end{array}$ & $\begin{array}{l}57.65 \pm 8.78 \\
(25-74)\end{array}$ & $\mathrm{NA}$ \\
\hline MMSE [0-30] & $24.4 \pm 1.9(21-30)$ & $27.9 \pm 1.1(27-30)$ \\
\hline $\begin{array}{l}\text { Anti-MAGlgM titer [ng/mL], } \\
\text { mean } \pm S D(\text { range) }\end{array}$ & $\begin{array}{l}2.66 \pm 1.57 \\
(0.28-8.87)\end{array}$ & $\begin{array}{l}1.87 \pm 1.48 \\
(0.31-9.30)\end{array} \quad \mathrm{p}<0.05$ \\
\hline
\end{tabular}

Table 2. Biochemical characteristic in a group ofPD patients (Mild/ moderate versus advanced Parkinson's disease)

\begin{tabular}{|c|c|c|c|}
\hline & \multicolumn{2}{|c|}{ PD patients } & $\mathrm{p}$ \\
\hline Hoehn-Yahr scale & $\begin{array}{l}\text { Early/moderate } \\
\text { PD patients } \\
\text { (Hoehn-Yahr 1-3) } \\
\mathrm{n}=42\end{array}$ & $\begin{array}{l}\text { Advanced PD } \\
\text { patients } \\
\text { (Hoehn-Yahr 4-5) } \\
n=24\end{array}$ & \multirow[t]{2}{*}{$p>0.05$} \\
\hline $\begin{array}{l}\text { Anti-MAG IgM antibodies levels } \\
{[\mathrm{ng} / \mathrm{mL}]} \\
\text { Median (range) }\end{array}$ & $\begin{array}{l}1.35 \\
(0.31-4.79)\end{array}$ & $\begin{array}{l}1.79 \\
(0.32-4.80)\end{array}$ & \\
\hline
\end{tabular}

Table 3. Rho Spearman correlation coefficients between anti-MAG IgM antibodies titers and clinical variables (stage of the PD on Hoehn-Yahr scale, MMSE and age of PD onset) in a group of PD patients.

MMSE - Mini Mental State Examination

\begin{tabular}{lcc}
\hline Clinical variable & Anti-MAG antibodies titers & $p$ \\
\hline Stage of disease on Hoehn-Yahr scale & 0.149 & 0.23 \\
\hline MMSE & 0.062 & 0.62 \\
\hline Age of PD onset & -0.042 & 0.74 \\
\hline
\end{tabular}

mild/moderate disease (grade 1-3 on the Hoehn-Yahr scale; median: 1.35) ( $\mathrm{p}>0.05)$. No correlations were found between anti-MAG IgM antibody titers and the stage of disease measured on the Hoehn-Yahr scale, MMSE or age of PD onset $(\mathrm{p}>0.05)$. Demographical, clinical and biochemical characteristics of the study population are shown in Tables 1 and 2. Spearman's rho correlation coefficients are presented in Table 3.

\section{DISCUSSION}

In the presented study, evidence was found for an increased production of anti-MAG antibodies in PD patients. The study confirmed previously obtained results, but on a larger group of patients, and support the concept that immune response is activated in the course of different neurodegenerative disorders $[2,18,21]$. The presented study was an attempt to assess IgM anti-MAG response in a larger group of PD patients, and also to assess its relation to disease stage. The data confirm that humoral response against MAG is significantly higher in PD subjects in comparison to healthy subjects. 
It is possible that as the neurodegenerative process spreads and brain cells die, new and unknown antigens are presented to the immune system, which gives rise to a secondary adaptive humoral response. A model showing how the adaptive immune response may be involved in the pathogenesis and progression of neurodegenerative disorders has been presented by Monahan [1]. A long-lasting neurodegenerative process leads to the death of central nervous system (CNS) cells and presentation of their antigens to the immune system, which activates T and B cells. B cells and specific autoantibodies then enter the CNS across the damaged blood-brain barrier (BBB), produce cytokines which activate microglia, and release antibodies. This phenomenon causes further inflammation and subsequent cell death [1].

The results of the current study also show that anti-MAG antibodies are not useful as biomarkers of disease severity. Interestingly, no correlation was found between antibody titers and clinical stage of the disease measured on theHoehnYahr scale. Taking into consideration Monahan's hypothesis, one could expect differences in antibody titers among subgroups of patients with different stages of the disease. It is worth noting, however, that Burke et al. [23] did not find a correlation between the degree of neurodegeneration assessed by Braak staging [24] and clinical severity of PD. This could possibly explain the lack of correlations between antibody titers and disease severity measured on the Hoehn-Yahr scale. The results obtasined in the current studyare partially in line with the results recently published by Maetzler et al. [2] who detected comparable levels of autoantibodies against two other anti-myelin antigens(antiMOG, anti-MPB) in PD patients and healthy subjects, and found no correlations between the antibody titers and disease duration, disease severity assessed on the Hoehn-Yahr scale, or MMSE. Although the results of the current study argue against the potential role of anti-MAG IgM antibodies as biomarkers of PD severity, these antibodies can probably still serve as biomarkers of the neurodegenerative process present in PD. Possibly, they could serve as biomarkers of $\mathrm{PD}$ at the preclinical stage, as it cannot be excluded that PD patients have increased values of respective autoantibodies also at earlier preclinical disease stages.

Obviously, the influence on autoimmune response, of factors other than a chronic neurodegenerative process, cannot be excluded. There is evidence that these triggering factors may include different infectious agents, particularly viruses, which exert their effects by influencing the activity of HSPs [25]. This is the reason the authors of the presented study assessed the immune response against MAG in relation to healthy controls, and found significantly higher titers of antiMAG autoantibodies in subjects with a neurodegenerative disorder. Considering the fact that these two groups probably have comparable exposition to viral infections, and that subjects with any history of infections, blood transfusions and immunomodulatory treatment three months prior to inclusion in the study were excluded, it can still hypothesize that the chronic neurodegenerative process has its own impact on eliciting an immune response.

It is still unclear whether the presence of the investigated autoantibodies is a primary factor responsible for neurodegeneration, an immune response secondary to the spreading neurodegenerative process, or the least a probable option, the described antibodies are only present in different degenerative CNS disorders without playing any pathogenic role. Additionally, it is worth mentioning that the presence of small titers of anti-MAG autoantibodies in healthy subjects can be explained by a probable mild inflammatory process ongoing in the normal aging brain [26]. Data on significantly higher microglial activation in the brains of elderly nondemented patients have been reported by Overmyer et al. [26].

Further studies are necessary to assess the role of anti-MAG response in PD in relation to other different neurodegenerative disorders (e.g., atypical parkinsonism, vascular parkinsonism and different types of dementia).

\section{CONCLUSIONS}

The results of this study provide evidence for the activation of humoral response against glial-derived proteins in $\mathrm{PD}$, which also indirectly reflects oligodendroglial degeneration under in vivo conditions. It is possible that the response is secondary to cell death in the central nervous system, and may indicate the potential protective role of autoimmunity in maintaining the body's homeostasis.This protective role possibly involves clearance of abnormal proteins, the imbalance of which may lead to earlier brain degeneration. The findings of the presented study define possible directions for future research on autoantibodies against disease-related proteins, especially glial-derived antigens in PD.

\section{Acknowledgements}

The authors thank the Medical University of Lublin, Poland, for Grant No. 387/2015.

\section{REFERENCES}

1. Monahan AJ, Warren M, Carvey PM.Neuroinflammation and peripheral immune infiltration in Parkinson's disease: an autoimmune hypothesis. Cell Transplant. 2008; 17: 363-367.

2. Maetzler W, Berg D, Synofzik M, Brockmann K, Godau J, Melms A, et al. Autoantibodies against amyloid and glial-derived antigens are increased in serum and cerebrospinal fluid of Lewy body-associated dementias. J Alzheimers Dis. 2008; 26: 171-179.

3. Yanamandra K, Gruden M, Casaite Y, Meskys R, Forsgren L, MorozovaRoche L. a-Synuclein reactive antibodies as diagnostic biomarkers in blood sera of Parkinson's disease patients. PLoS 2011; 25: e18513.

4. Martino D, Church A, Giovannoni G. Are antibasal ganglia antibodies important, and clinically useful? Pract Neurol. 2007; 7: 32-41.

5. Berger T, Rubner P, Schautzer F, Egg R, Ulmer H, Mayringer I, Dilitz E, Deisenhammer F, Reindl M. Antimyelin antibodies as a predictor of clinically definite multiple sclerosis after a first demyelinating event. N Engl J Med. 2003; 349: 139-145.

6. Pihan M, Decaux O, Marcorelles P, Bahon-Riedinger I, Lemercier S, Gainche-Salmon A, Doncker AV. Neuropathies associated with monoclonal IgM anti-MAG antibodies. Rev Med Interne. 2012; 33: 686-92. doi: 10.1016/j.revmed.2012.05.009.

7. Magy L, Kaboré R, Mathis S, Lebeau P, Ghorab K, Caudie C, Vallat JM. Heterogeneity of Polyneuropathy Associated with Anti-MAG Antibodies. J Immunol Res. 2015: 450391. doi: 10.1155/2015/450391.

8. Maetzler W, Berg D, Synofzik M, Brockmann K, Godau J, Melms A, Gasser T, Hörnig S, Langkamp M. Autoantibodies Against Amyloid and Glial-Derived Antigens are Increased in Serum and Cerebrospinal Fluid of Lewy Body-Associated Dementias. J Alzheimers Dis. 2011; 26: 171-179.

9. Gruden MA, Davidova TB, Malisauskas M, Sewell RD, Voskresenskaya NI, WilhelmK et al. Differential neuroimmune markers to the onset of Alzheimer's disease neurodegeneration and dementia: autoantibodies to Abeta ((25-35)) oligomers, S100b and neurotransmitters. J Neuroimmunol. 2007; 186: 181-192.

10. Papachroni KK, Ninkina N, Papapanagiotou A, Hadjigeorgiou GM, Xiromerisiou G, et al. Autoantibodies to alpha-synuclein in inherited Parkinson's disease. J Neurochem. 20007; 101: 749-756. 
11. Besong-Agbo D, Wolf E, Jessen F, Oechsner M, Hametner E, et al. Naturally occurring alpha-synuclein autoantibody levels are lower in patients with Parkinson disease. Neurology 2013; 80: 169-75.

12. Wakabayashi K, Hayashi S, Yoshimoto M, Kudo H, Takahashi H. NACP/alpha-synuclein-positive filamentous inclusions in astrocytes and oligodendrocytes of Parkinson's disease brains. ActaNeuropathol. (Berl) 2000; 99: 14-20.

13. Ahmed Z, Asi YT, Lees AJ, Revesz T, Holton JL. Identification and quantification of oligodendrocyte precursor cells in multiple system atrophy, progressive supranuclear palsy and Parkinson's disease. Brain Pathol. 2013; 23: 263-273.

14. Papuć E, Kurzepa J, Kurys-Denis E, Grabarska A, Krupski W, Rejdak K. Humoral response against glial derived antigens in Parkinson's disease. Neurosci Lett. 2014; 566: 77-81.

15. Maetzler W, Apel A, Langkamp M, Deuschle C, Dilger SS, Stirnkorb JG, et al. Comparable autoantibody serum levels against amyloid-and inflammation associated proteins in Parkinson's disease patients and controls. PLoS One 2014; 21:e88604. doi: 10.1371/journal.pone.0088604.

16. Koehler NK, Stransky E, Shing M, Gaertner S, Meyer M, et al. Altered Serum IgG Levels to alpha-Synuclein in Dementia with Lewy Bodies and Alzheimer's Disease. PLoS One 2013; 8: e64649.

17. Papuć E, Kurys-Denis E, Krupski W, Tatara M, Rejdak K. Can Antibodies Against Glial Derived Antigens be Early Biomarkers of Hippocampal Demyelination and Memory Loss in Alzheimer's Disease? J Alzheimers Dis. 2015; 28: 115-121. doi: 10.3233/JAD-150309.

18. Nguyen T, Mehta NR, Conant K, Kim KJ, Jones M, Calabresi PA, et al. Axonal protective effects of the myelin associated glycoprotein. J Neurosci. 2009; 29: 630- 637.
19. Thompson HJ, Marklund N, LeBold DG, Morales DM, Keck CA, Vinson $\mathrm{M}$, et al. Tissue sparing and functional recovery following experimental traumatic brain injury is provided by treatment with an anti-myelinassociated glycoprotein antibody. Eur J Neurosci. 2006; 24: 3063-3072.

20. Irving EA, Vinson M, Rosin C, Roberts JC, Chapman DM, Facci L, et al. Identification of neuroprotective properties of anti-MAG antibody: a novel approach for the treatment of stroke? J Cereb Blood Flow Metab. 2005; 25: 98-107.

21. DeMattos RB, Bale KR, Cummins DJ, Dodart JC, Paul SM, Holtzman DM. Peripheral anti-A beta antibody alters CNS and plasma A beta clearance and decreases brain A beta burden in a mouse model of Alzheimer's disease. Proc Natl Acad Sci. USA 2001; 98: 8850-8855.

22. Postuma RB, Berg D, Stern M, Poewe W, Olanow CW, Oertel W, et al. MDS clinical diagnostic criteria for Parkinson's disease. Mov Disord. 2015; 30: 1591-1601. doi: 10.1002/mds.26424.

23. Burke RE, Dauer WE, Vonsattel JP. A critical evaluation of the Braak staging scheme for Parkinson's disease. Ann Neurol. 2008; 64: 485-491. doi: 10.1002/ana.21541.

24. Braak H, Del Tredici K, Rüb U, de Vos RA, Jansen Steur EN, Braak E. Staging of brain pathology related to sporadic Parkinson's disease. Neurobiol Aging. 2003; 24: 197-111.

25. Temajo NO, Howard N. The virus-induced HSPs regulate the apoptosis of operatus APCs that results in autoimmunity, not in homeostasis. See comment in PubMed Commons below Autoimmun Rev. 2014; 13: 1013-1019.

26. Overmyer M, Helisalmi S, Soininen S, Laakso M, Riekkinen P, Alafuzoff I. Reactive microglia in aging and dementia: an immunohistochemical study of postmortem human brain tissue, Acta Neuropathol. (Berl) 1999; 97: 383-392. 